\title{
Investigation of Heat Transfer of Tube Line of Staggered Tube Bank in Two- Phase Flow
}

\section{Mindaugas Jakubcionis}

European Commission, Joint Research Centre, Institute for Energy and Transport, P.O. Box 2, NL-1755ZG Petten, The Netherlands

(C) Science Press and Institute of Engineering Thermophysics, CAS and Springer-Verlag Berlin Heidelberg 2015

\begin{abstract}
This article presents the results of experimental investigation of heat transfer process, carried out using the model of heat exchanger. Two-phase statically stable foam flow was used as a heat transfer fluid. Heat exchanger model consisted of staggered tube bank. Experimental results are presented with the focus on influence of tube position in the line of the bank, volumetric void component and velocity of gas component of the foam. The phenomena of liquid draining in cellular foam flow and its influence on heat transfer rate has also been discussed. The experimental results have been generalized by relationship between Nusselt, Reynolds and Prandtl numbers.
\end{abstract}

\section{Keywords: two phase flow, heat transfer, heat exchanger}

\section{Introduction}

The simplest case of material flow is homogeneous one-phase flow in which its components are mixed in molecular level. Two-phase or heterogeneous flow, on the other hand, is a peculiar case of material flow. It occurs when flow consists of two components with different state, for instance liquid and gas or liquid and solid. Two-phase flows consisting of liquid and gas are found in many technological processes dealing with heat and mass transfer. Such processes are used in a wide range of applications in many fields of engineering and science such as mechanical engineering, nuclear engineering, energy engineering, chemical engineering and so on.

The need to preserve the environment and rising energy costs are the main driving forces in development of new and more efficient methods and means of energy generation. Naturally, technological requirements and possibilities for wider utilization of two-phase flow apparatus should not be overlooked also.

In modern technologies different class of heterogene- ous flows - dispersive flows - is founding increasingly wider application. Dispersive flows are made of mixtures of different phases, namely foam, bubbles, droplets of liquid or solid particles.

Foam is formed when liquid and gas flows are coming into contact. Depending on the structure and properties foam can be divided into different groups: turbulized dynamic foam, structural foam and statically stable foam ${ }^{[1]}$.

Statically stable foam can be used in evaporation, concentration, drying and combustion processes as well as in a variety of technological systems for wastewater treatment and utilization ${ }^{[1]}$. Due to their small dimensions, low energy and material consumption foam utilizing apparatus can be suitable to use in heat exchangers, food industry, chemical and oil processing industry. Such heat exchangers could be applied in cases when there is a need to transfer relatively moderate amount of heat when water cooling would be too intense but gas cooling would be insufficient. Heat transfer coefficient of homogeneous water flow at $0.4 \mathrm{~m} / \mathrm{s}$ velocity would be approximately $4000 \mathrm{~W} / \mathrm{m}^{2} \mathrm{~K}$ while heat transfer coefficient 
of air under the same conditions would be only 16 $\mathrm{W} / \mathrm{m}^{2} \mathrm{~K}$. Statically stable foam, while consisting of water and air, is neither of the two and its properties are quite different from its components. Experimental investigations have shown that heat transfer of statically stable foam under above mentioned conditions can range in the area of $400-1000 \mathrm{~W} / \mathrm{m}^{2} \mathrm{~K}^{[2]}$ depending on volumetric void faction of foam flow.

Heat transfer of pipes and tube bundles in laminar flow of foam has been investigated by different authors ${ }^{[3,4]}$. Scientific research in this area mainly includes particular problems like utilization of statically stable foam flow apparatus or investigation of particular foam flow properties $^{[5]}$. In some cases conclusions and results of such theoretical investigations can be applied to the layer of statically stable foam flow only and are not suitable to use when dealing with processes taking part in foam flow.

In recent years quite a number of investigations have been performed while dealing with heat transfer of tubes and their bundles in foam flow ${ }^{[6]}$. But in order to use statically stable foam in design and operation of heat exchangers engineers should have generalized and easy to use data on tendencies of heat transfer coefficient dependence on foam flow velocity and other influencing factors which was lacking up until now.

\section{Foam formation and flow patterns}

The flow pattern of two-phase flow changes while it goes through the channel. At first when liquid solution comes into contact with streams of air, bubbles are formed. Bubbles agglomerate and form at first dynamically stable and then statically stable foam. Dynamically stable foam consists of turbulent flow made of two phases - gas and liquid. Despite its name dynamically stable, turbulent foam is a very unstable system which exists only during immediate gas and liquid contact. If the gas flow is interrupted turbulent foam decompounds into pure liquid and gas right away (in case of foam generation from pure liquid) or it turns into statically stable foam (in case detergent solution was used). Dynamically stable foam boasts high values of heat transfer coefficient but it is notoriously difficult to use it in controlled manner.

It is impossible to create statically stable foam using pure liquid. It can only be formed from detergent solution. Statically stable foam is made of gas bubbles in the shape of regular polygons, separated by thin liquid films. Thus this kind of foam is also called cellular foam. Statically stable foam, contrary to dynamically stable foam, lives perfectly up to its name since in some cases it can last for quite a long time, spanning to days and even years.

Another method to define the structure of different foam types is their division into so-called wet and dry foams. Wet foams are made of spherical bubbles while dry foam is made of polyhedral bubbles.

Statically stable foam can be made using liquid solutions with decreased surface tension. Such reduction in surface tension can simply be achieved by addition of detergents into pure liquid, for instance water. Even the addition of small amount of detergent results in intense statically stable foam generation when gas streams pass through the layer of liquid solution. Minimal concentration of detergents which is sufficient for transition of solution from liquid state to statically stable foam is different for different kind of liquids and detergent materials. Experimental investigations ${ }^{[2]}$ showed that such minimal concentration of detergents is equal to $0.10 .3 \%$. The exact value of minimal concentration depends on different factors, such as gas velocity, height of foam column, temperature of liquid and so on.

The frame of statically stable foam flow consists of liquid films which are almost flat and which at the same time act as the walls of foam bubbles. At the place where three films come into contact foam bubbles are conjoined at $120^{\circ}$ angle as it is shown in Figure 1. In the place of conjunction a so called Plateu channels are formed and there liquid has a concave surface in which liquid pressure is significantly lower than in the flat films. Due to the pressure difference liquid flows from the walls of foam frame to Plateu channels and foam walls become thinner. It shoud be noted that the deposition of liquid through Plateu channels is a relatively slow process but it nevertheless has significant influence on heat transfer processes taking part in foam flow.

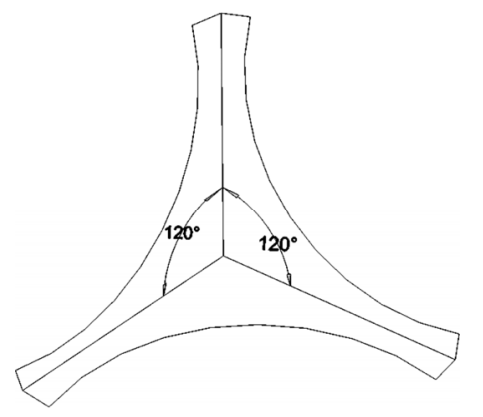

Fig. 1 Structure of plateu channels of foam bubbles

Experimental investigations ${ }^{[2]}$ showed that there are four distinct regimes of statically stable upward foam flow in the vertical channel: Laminar foam flow $\left(\overline{\mathrm{Re}}_{g}=\right.$ $0 \div 600$ ), Transitional foam flow $\left(\overline{\operatorname{Re}}_{g}=600 \div 1500\right)$, Turbulent foam flow $\left(\overline{\operatorname{Re}}_{g}=1500 \div 1900\right)$ and Emulsion foam flow $\left(\overline{\operatorname{Re}}_{g}>1900\right)$. As experimental investigations, whose results are presented in this paper showed, these 
numbers are to be used with caution because limits of foam flow regimes clearly depend on many factors, among them different cross section of foam column which was somewhat overlooked in investigation ${ }^{[2]}$. It has been noticed that already at Reynolds number of 500 clear attributes of transitional regime are noticeable, among others pulsations and instability of foam flow. This clearly demonstrates the complexity of processes taking part in foam flow and emphasizes the caution which should be used while describing them and making solid conclusions.

\section{Experimental method and procedure}

In order to determine heat transfer rate of tube bundle in statically stable foam flow, experimental arrangement was constructed. Experimental model consisted of the following main parts: vertically arranged experimental channel at the bottom of which foam was generated, reservoirs for storage of prepared liquid solution, air fan for delivery of gas component of the foam, electric power source for heating of experimental tube and measuring equipment for controlling and registering of liquid and gas flow rates and temperature changes.

Experimental channel consisted of the riddle at the bottom of it and experimental section. The cross section of the channel was equal to $0.18 \times 0.18 \mathrm{~m}^{2}$. Foam was generated on the riddle from liquid solution layer which was being penetrated by air streams coming through openings of the riddle. Liquid solution was used only once and was discarded after leaving experimental channel. Concentration of surfactants was kept constant and was equal to $0.5 \mathrm{wt} \%$.

Experimental section consisted of staggered tube bank, the scheme of which is presented in Figure 2.

Tube bundle consisted of eighteen tubes arranged in five rows with six tubes in each. The external diameter of the tubes was equal to $0.02 \mathrm{~m}$. The transverse pitch $S_{T}$ and longitudinal pitch $S_{L}$ between the centers of the tubes of experimental tube bank was equal to $S_{T}=S_{L}=0.035 \mathrm{~m}$. The heated tube was also made of copper as it was heated using electric current. The endings of the tubes were insulated to prevent heat losses through them. Two side rows of the tubes (one on each side) have only been used as dummies to simulate the arrangement of real life heat exchanger.

The temperature of foam flow and heated surface was measured using calibrated thermocouples. Temperature of heated surface was measured using eight thermocouples arranged around the central perimeter of the heated tube.

Investigation of tube heat transfer in the bundle consisted of three series of experiments. The experiments were performed for different values of mean volumetric void component $\varphi=0.996,0.997$ and 0.998 .

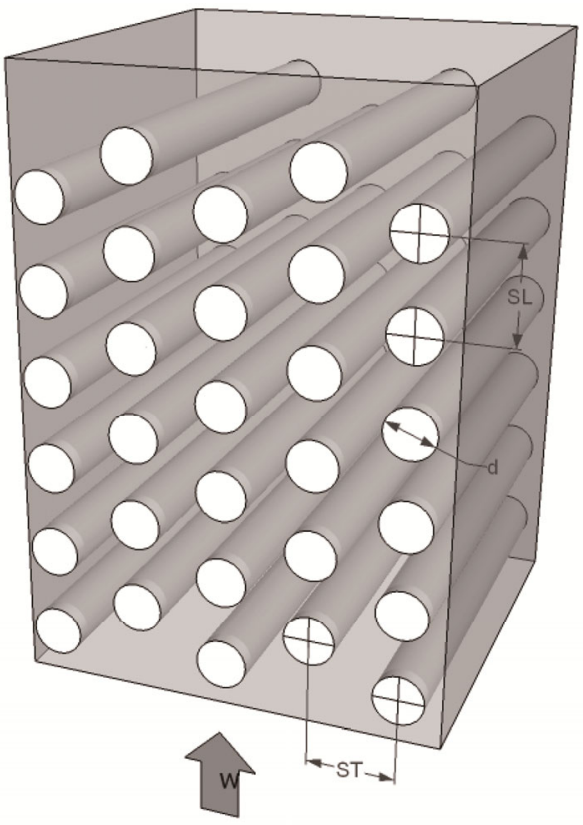

Fig. 2 Scheme of experimental tube bank

The volumetric void component can be expressed by equation:

$$
\varphi=V_{g} /\left(V_{g}+V_{l}\right)
$$

here $V_{g}$ - volumetric flow rate of gas component, $\mathrm{m}^{3} / \mathrm{s} ; V_{l}$ - volumetric flow rate of liquid component.

It should be stressed that value of volumetric void component was being kept constant only during statically stable foam formation, i.e. at the bottom part of experimental channel. In experimental section of the channel real void component values might have been quite different due to the breaking of larger foam particles into smaller ones containing larger amounts of liquid solution on their walls and other factors.

The volumetric foam flow rate can be expressed as follows:

$$
V_{f}=V_{g}+V_{l}
$$

Superficial gas and liquid velocities were calculated according to the changes in volumetric liquid and gas component rates:

$$
\begin{gathered}
w_{g}=V_{g} / A \\
w_{l}=V_{l} / A
\end{gathered}
$$

here $A$ - cross-section area of the experimental channel in most obstructed part, $\mathrm{m}^{2}$.

The experiments consisted of measurements of heated surface and foam flow temperature changes influenced by the changes in statically stable foam rate. All temperature values were registered after expiring of $90 \mathrm{sec}$. period after the last changes in foam flow rate of electric current since observations showed that the laminar foam flow regime becomes stable in experimental channel after such a period of time. After registration of measured values the temperature difference $\overline{\Delta T}$ between mean foam 
temperature and mean heated surface temperature was calculated. After calculation of heat flux density on the heated surface $q_{w}$ the average heat transfer coefficient was calculated as follows:

$$
\bar{\alpha}=q_{w} / \Delta T
$$

Experimental results have been generalized using dependence between average Nusselt, Reynolds and Prandtl numbers:

$$
\overline{N u}_{f}=f\left(\overline{\operatorname{Re}}_{g} \cdot \overline{\operatorname{Pr}}_{f}\right)
$$

In this dependence Nusselt number was calculated as follows:

$$
\overline{N u}_{f}=\frac{\bar{\alpha} \cdot d_{e}}{\lambda_{f}}
$$

Since statically stable foam flow is a two-phase flow, consisting mainly of gas volumes separated by thin films of liquid, it is difficult to determine actual thermal conductivity. In general heat conduction of foam flow is low and it was calculated using formula ${ }^{[1]}$ :

$$
\bar{\lambda}_{f}=\varphi \cdot \lambda_{l}+(1-\varphi) \cdot \lambda_{g}
$$

here $\lambda_{l}$ - thermal conductivity of liquid component, $\mathrm{W} / \mathrm{m}^{2} \mathrm{~K} ; \lambda_{g}-$ thermal conductivity of gas component, $\mathrm{W} / \mathrm{m}^{2} \mathrm{~K}$.

Reynolds number was calculated using formula:

$$
\overline{\operatorname{Re}}_{g}=\frac{\bar{w}_{g} \cdot d}{\bar{v}_{f}}
$$

here $\bar{w}_{g}$ - average specific velocity of gas component, $\mathrm{m} / \mathrm{s}, v_{f}$ - kinematic viscosity of foam flow, $\mathrm{m}^{2} / \mathrm{s}$.

Prandtl number has been calculated as follows:

$$
\operatorname{Pr}=\frac{P e}{\operatorname{Re}}=\frac{v}{a}=\frac{\mu_{f} \cdot c_{p l}}{\lambda_{f}}
$$

here $\mu_{f}$-dynamic viscosity of foam flow, $\mathrm{Pa} \cdot \mathrm{s} ; c_{p l}-$ specific heat of liquid component, $\mathrm{J} / \mathrm{kgK}$.

\section{Heat transfer of tube bank}

Experimental investigations of tube bank heat transfer in statically stable foam flow have been performed using three different values of volumetric void faction $\varphi=$ 0.994, 0.996 and 0.998 . The results of experiments for the first tube of the middle row of the bank are presented in Figure 3.

The experimental data shows that heat transfer rate is highly dependent on the average gas velocity and volumetric void faction of the foam flow. Values of heat transfer coefficient increase with the increase of gas velocity and with the decrease of volumetric void faction. The higher gas velocity, the more turbulent foam flow becomes and more intense destruction of larger gas bubbles takes place when foam flow comes into contact with solid surfaces of tubes in the bank. This leads to the in- crease of liquid component concentration in boundary layer on heated surface and more intense destruction of this layer. When the effects of higher gas velocity and reduced void faction are combined, the resulting effect on heat transfer rate is much more significant. The character of dependencies is similar for all the tubes constituting tube bank, but the rate of heat transfer increase is somewhat different.

These effects can clearly be seen from the curves presented in Figure 3.

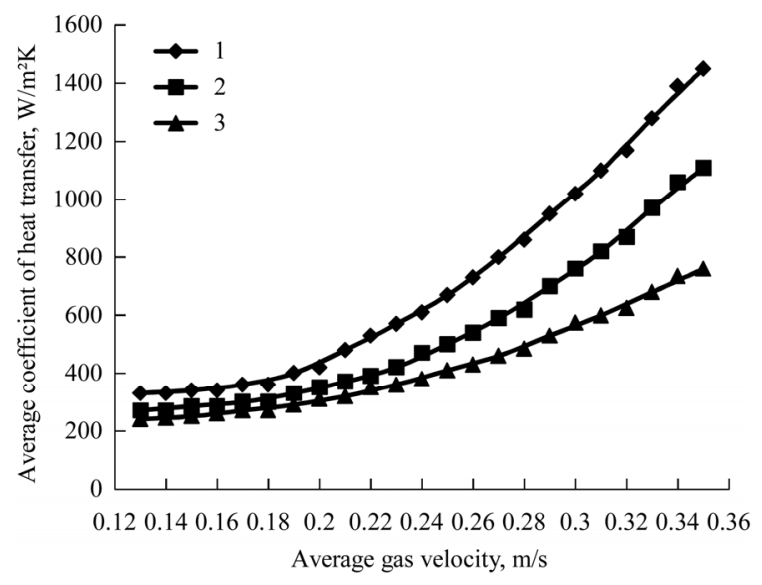

Fig. 3 Heat transfer rate of the first tube in the line of a staggered tube bank.1: $\varphi=0.994 ; 2: \varphi=0.996 ; 3: \varphi=$ 0.998 .

In the interval of analyzed gas velocity $\bar{w}_{g}=0.14 \div$ $0.35 \mathrm{~m} / \mathrm{s}$ heat transfer rate of the first tube in the line more than quadrupled, i.e. it increased from 330 till 1450 $\mathrm{W} / \mathrm{m}^{2} \mathrm{~K}$. Meanwhile for the fourth tube in the line heat transfer rate was trebled, i.e. it increased from 320 till $930 \mathrm{~W} / \mathrm{m}^{2} \mathrm{~K}$. The results of experiments for the fourth tube of the middle row of the bank are presented in Figure 4 .

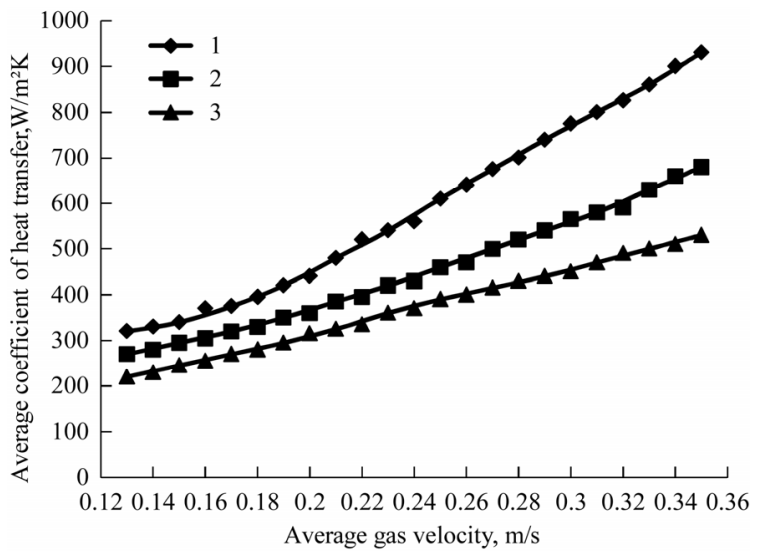

Fig. 4 Heat transfer rate of the fourth tube in the line of a staggered tube bank.1: $\varphi=0.994 ; 2: \varphi=0.996 ; 3: \varphi=$ 0.998 . 
Such dependencies are different in comparison with heat transfer of tube bank in one-phase flows. In case of homogeneous flow heat transfer rates of the first and the second tube in the staggered tube bank make up only a part of heat transfer rate of the third tube in the bank (approximately 60 and $70 \%$ correspondingly ${ }^{[7]}$. As we can see in Figures 3 and 4 initially heat transfer rate of the first and the fourth tubes is similar, but starting from gas velocity of $0.24 \mathrm{~m} / \mathrm{s}$ heat transfer rate of the first tube increases at much higher pace. The reason of such dependencies can be explained by the fact that the first tube of the bank is affected by direct foam flow. Statically stable foam flow consists of gas volumes which are more easily broken into smaller ones when foam comes into contact with solid surface of tubes in the bank. Smaller gas volumes mean larger local concentration of liquid films constituting walls of the bubbles and, correspondingly, more favorable heat transfer conditions. The following tubes are located in limited internal space of the bank where foam flow loses its energy and local volumetric void faction is higher in comparison with frontal row of the tubes.

In Figure 5 the experimental results of heat transfer for the tubes of the middle row of bank are plotted against gas velocity. The results which are presented in this figure have been obtained while volumetric void faction of statically stable foam flow was kept at 0.996 .

As it can be seen in this figure in initial period of gas velocity augmentation $\left(\bar{w}_{g}=0.14 \div 0.24 \mathrm{~m} / \mathrm{s}\right)$ heat transfer rate is almost independent of tube position in the line exception being heat transfer rate of the sixth tube which is significantly higher than that of the other tubes.

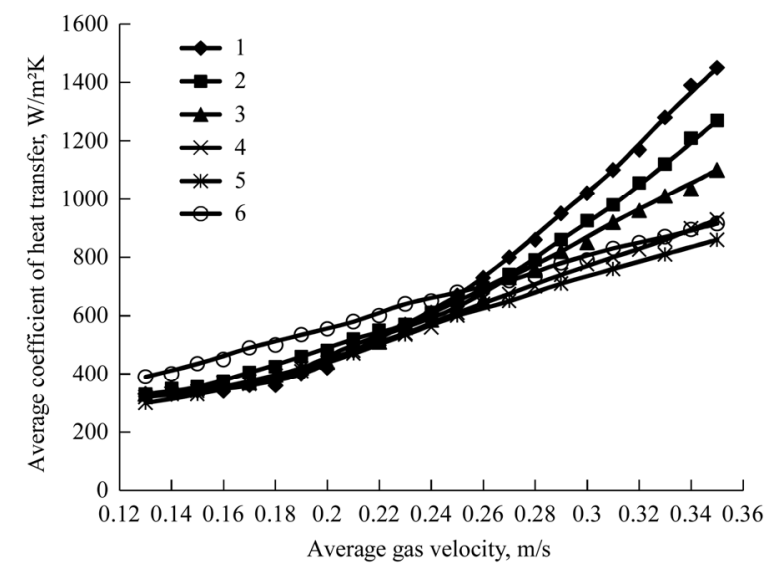

Fig. 5 Heat transfer rate of the tube line of a staggered tube bank. Numbers correspond to the position of the tube in the line

It can be explained due to the changes in foam structure which occur while foam passes through tube bank. When gas velocity is low, foam, which is formed at the bottom part of experimental channel, consists of large bubbles $\left(d_{b} \cong 8 \div 14 \mathrm{~mm}\right.$ ). The structure of statically foam flow in the bottom part of experimental channel at low flow velocity can be seen in Figure 6a. When foam passes through tubes of the bank its bubbles brake into smaller ones $\left(d_{b} \cong 2 \div 5 \mathrm{~mm}\right)$. In such a way a layer of small structure foam is formed above tube bank. This layer is characterized not only by decreased volumetric void faction but also by more intense processes of liquid drainage from foam walls and more intense turbulization.

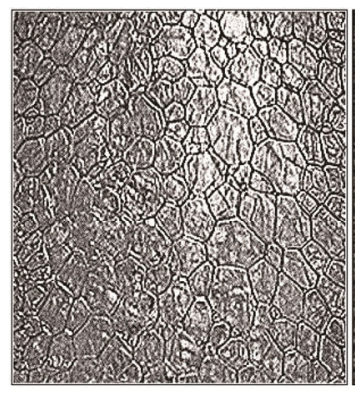

(a)

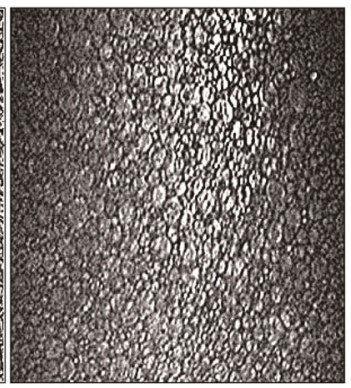

(b)
Fig. 6 Structure of statically stable foam flow before experimental part of test channel; $\varphi=0.994$; a) $\bar{w}_{g}=0.17$ $\mathrm{m} / \mathrm{s} ; \mathrm{b}) \quad \bar{w}_{g}=0.34 \mathrm{~m} / \mathrm{s}$.

When gas velocity increases above $0.24 \mathrm{~m} / \mathrm{s}$ the foam which is generated in the bottom part of experimental channel already consists of smaller bubbles as it shown in Figure $6 \mathrm{~b}$ and most intense heat transfer rate comes in case of the first tube of the line since conditions of heat transfer in initial part of the bank become increasingly more favorable. In this period of gas velocity augmentation heat transfer rate of the sixth tube in the line becomes similar to heat transfer rate of the third and following tubes.

\section{Heat transfer generalization}

The experimental results of heat transfer rate dependence on gas velocity have been generalized using dependence between Nusselt, Reynolds and Prandtl numbers (6).

The correlation for the heat transfer of tube line of the bank in statically stable foam flow can be written as follows:

$$
\overline{N u}_{f}=C \overline{\operatorname{Re}}_{g}^{m} \operatorname{Pr}_{f}^{1.5}\left(\frac{\operatorname{Pr}_{f}}{\operatorname{Pr}_{w}}\right)^{1.2}
$$

Here all properties, except $P r_{s}$ are evaluated at the arithmetic mean of the foam temperature before and after tube bank.

For the interval of Reynolds number $180<\overline{\mathrm{Re}}_{g}$ 
$<300, C=20$ and $\mathrm{m}=0.4$.

For the interval of Reynolds number $300<\overline{\mathrm{Re}}_{g}$ $<500, \mathrm{~m}=1.6$. Values of correction factor $C$ for this interval of Reynolds number, depending on the position of tube in the line $N_{L}$, are presented in Table 1 .

Table 1 Correction factor $C$ of Equation 12

\begin{tabular}{ccccc}
\hline$N_{L}$ & $1-3$ & 4 & 5 & 6 \\
$C$ & 0.0210 & 0.0244 & 0.0265 & 0.0272 \\
\hline
\end{tabular}

Heat transfer rate of the last tube of the line (in this instance it was the sixth tube) has not been generalized since it clearly does not correspond to the general tendencies observed in cases of other tubes of the bank.

\section{Conclusions}

The results of experimental investigation of heat transfer from the line of tubes of staggered tube bank to cross flowing statically stable foam flow have been presented. The heat transfer coefficients have been determined for different foam velocities and different volumetric void components of the foam.

Heat transfer rate of tubes in foam flow increases with an increase of specific gas velocity and decrease of volumetric void component. Heat transfer rate increase in tube bank is highly uneven and the highest was observed in the case of the first tube in line and its character becomes increasingly flat further into the bank.

Experimental results of heat transfer investigation have been generalized using dependence between Reynolds, Nusselt and Prandtl numbers.

\section{References}

[1] Tichonov, V., Theory and Practice of Foam Generation and Destruction, Chemistry, Moscow, 1996 (in Russian)

[2] Gylys, J., Hydrodynamics, Heat and Mass Transfer under the Cellular Foam Systems, Technologija, Kaunas, 1998 (in Lithuanian)

[3] Gylys, J., Zdankus, T., Miliauskas G. and Sinkunas., S, Influence of Vertical Foam Flow Liquid Drainage on Tube Bundle Heat Transfer Intensity, Experimental Heat Transfer: A Journal of Thermal Energy Generation, Transport, Storage, and Conversion, Vol.20, Taylor and Francis, 2007.

[4] Attia, J.A., Mckinley, I.M., Moreno-Magana, D., Pilon, L. Convective heat transfer in foams under laminar flow in pipes and tube bundles. International Journal of Heat and Mass Transfer, Vol.55, p.p. 7823-7831, Elsevier, 2012.

[5] Fournel, B., Lemonnier, H. and Pouvreau, J. Foam Drainage Characterization by Using Impedance Methods. Proc. 3rd International Symposium on Two-Phase Flow Modelling and Experimentation. pp.1-7. Pisa, Italy, 2004.

[6] Gylys, J., Jakubcionis, M., Sinkunas, S. and Zdankus, T. An Experimental Study of Upward and Downward Foam Flow in Small Test Bundle. Proc. 12th International Heat Transfer Conference. Grenoble, France. pp.399-404, 2002.

[7] Zukauskas, A., Heat Transfer from Tubes in Cross Flow, Advances in Heat Transfer, Vol. 8, Academic Press, New York, 1972. 